\title{
Justification of using Computed Tomography and Magnetic Resonance Imaging for Deep Venous Thrombosis and Pulmonary Embolism
}

\author{
https://doi.org/10.3991/ijoe.v17i14.26577 \\ Petro Bodnar ${ }^{(\bowtie)}$, Yaroslav Bodnar, Tetiana Bodnar, Liudmyla Bodnar, \\ Dymytriy Hvalyboha \\ I. Horbachevsky Ternopil National Medical University, Ternopil, Ukraine \\ Bodnarpj@tdmu.edu.ua
}

\begin{abstract}
Deep vein thrombosis (DVT) is a medical condition, occurs when a blood clot forms in a deep vein and pulmonary embolism (PE) occurs when a blood clot gets lodged in an artery in the lung, affecting blood flow to part of the lung.The frequencies of using computed tomography (CT) and magnetic resonance imaging (MRI) to diagnose deep venous thrombosis and pulmonary embolism is increasing day by day.

Both the technics are noninvasive and provide prompt results. But there are a good number of alternative technics for the same purposes. That is why, till now scholars and respective professionals are interested to know more about the justification and comparative effectiveness of CT and MRI in detecting DVT and PE.

This review aimed to analyze the history of several detecting methods for DVT and PE and to dig out the clear concepts about the effectiveness and patient compliances of $\mathrm{CT}$ and MRI in detecting deep venous thrombosis and pulmonary embolism. For proper analysis a lot of research as well as meta-analysis had been studied.

From this article besides scholars and professionals, general readers will get a clear concept about the features, effectiveness and justifications of CT and MRI in treating DVT and PE.
\end{abstract}

Keywords — computed tomography (CT), magnetic resonance imaging (MRI), deep vein thrombosis, pulmonary embolism (PE), angiography

\section{Introduction}

\subsection{Deep vein thrombosis (DVT)}

Deep vein thrombosis (DVT) happens when a blood clot forms in one or more of the deep veins in the human body, which can affect the blood flow of veins and result in poor blood circulation. Moreover, DVT can occur even in the absence of serious disorders. As investigated by some researchers, 'thrombosis can occur in any part of the venous system, but it appears most frequently at the deep veins of the legs' [1].

Thrombosis can occur during travelling and even that can be in an air traveler also. 'Traveler's thrombosis occurs by sitting still in transport-vehicle for a long time and 
blood clots are formed and deposited the inside of deep veins of lower limbs', which constitutes DVT [2]. Scurr et al. have indicated 'incidences of $10 \%$ of asymptomatic calf vein thrombosis among air travelers $>50$ years of age and traveling for more than 8 h' [3]. The prevalence of DVT among the general population and the possibilities of the suffering of DVT patients from pulmonary embolism is not so small. Basically, 'DVT is a prevalent disease in nature with an occurrence of between ' $2.5 \%$ to $5 \%$ ' in the total population, and about ' $30-40 \%$ ' of DVT patients finally suffer from 'pulmonary embolism' [4].

A great disappointing thing is the uncertainty of proper diagnostic results of DVT. Generally, of DVT is a great clinical challenge for doctors and clinicians. Besides these, the deterioration of such disease may make the treatment procedure more complicated. Actually, 'the diagnosis or assessment of DVT in clinics is notoriously unreliable because, only about $30 \%$ patients have been shown to be positive on objective testing' [5]. That is why, 'suspected DVT cases in the clinical diagnosis, can lead to the 'unnecessary prolonged hospitalization of patients' as well as 'inappropriate anticoagulation therapy' with potentially harmful and dangerous consequences.

\subsection{Pulmonary embolism (PE)}

Pulmonary embolism is a common but still underdiagnosed condition. Pulmonary embolism (PE), which is the most serious presentation of VTE, is the third most common cause of death from cardiovascular disease after a heart attack and stroke. [6] In a survey, it was found that, "1945 through 2002, PE was unsuspected or undiagnosed ante-mortem in 3268 (84\%) of 3876 patients" who had PE discovered at autopsy [7].

It is still undiagnosed, due to the lack of suspicion and availability of appropriate diagnostic testing such as multi-detector computed tomographic angiography (CTA), especially in low resource settings [8]. It is so serious condition that has a '30-day mortality rate' between $9 \%$ and $11 \%$ [9]. Worldwide, the prevalence of PE is increasing day by day which is most alarming. The incidence of PE (Pulmonary embolism) is growing worldwide, particularly in the high-income countries [10].

Defining PE depending on clinical presentations is very critical and uncertain. Generally, the clinical features of pulmonary embolism are non-specific. In a study, they claimed that, 'Symptoms can vary from mild chest pain to shock be-cause of right ventricular failure in patients with massive PE' [11]. In a study they claimed, 'patients with PE sometimes present without any symptoms, the diagnosis being coincidently when investigating for other conditions [10]'.

Till now, spinal cord injury, immobilization, malignancy, recent surgery and/or trauma, lower limb fractures and joint replacements are considered as the major risk factors of PE. However, 30\% of patients with PE have no detectable provoking factors [12] and 'in Sub-Saharan Africa, preliminary studies have shown that PE was scarce' [13]. 


\section{Methodology}

Taking into account the specifics of the topic under consideration, the most appropriate when writing this article was the use of the a systematic review of the literature was developed in three main processes, considering the criteria. The established criteria are the following: research planning, development of the search for information, and results found.

\section{Diagnosis of deep vein thrombosis and pulmonary embolism}

The diagnosis of pulmonary embolism (PE) is a very difficult task, particularly when there is coexisting 'heart or lung disease' and it is notoriously inaccurate when based on clinical signs alone. Sometimes, pulmonary embolism manifests in such a dramatic fashion that, the prompt diagnosis is intuitively obvious and treatment will be started, but the usual presentation is frequently 'vague' and variable in severity and further testing is necessary to establish or exclude the diagnosis [14]. About all the patients of pulmonary embolism will have 'one or more' of dyspnea of sudden onset, tachypnoea (20 breaths per min), or chest-pain (pleuritic/substernal) [15], if the clinician remembers those 3 features, the possibility of pulmonary embolism (PE) will rarely be overlooked. While these clinical features and/er presentations are associated with 'electrocardiographic signs of right ventricular strain' and/or 'radiological signs of plump hilum', pulmonary infarction or oligemia, the likelihood of PE is high and it is further strengthened in the presence of 'risk factors for VTE' and arterial hypo-anemia with hypocapnia [14]. But, the absence of all these 3 clinical features/presentations, virtually excludes the diagnosis of PE (Pulmonary embolism) [16]. Different studies have disseminated that, well characterized clinical empiric estimates or explicit 'prediction rules of pretest likelihood of PE can be applied for the safe management of patients suspected of having the disease [17]. Year after year many diagnostic methods for detecting pulmonary embolism have been introduced in the treatment arena.

\subsection{Several diagnostic methods of DVT and PE}

There is a tradition of using several diagnostic tools and methods in detecting pulmonary embolism (PE) as well as deep vein thrombosis (DVT). Some are used as independent and some as associate. Here is an overview of several diagnostic tools and methods of PE and DVT.

Electrocardiography. Electrocardiography is a primary diagnostic method for pulmonary embolism. The main value of electrocardiography (ECG) is in excluding other potential diagnoses like myocardial infarction or pericarditis [14]. Generally, in minor pulmonary embolism (PE), there is no real hemodynamic stress and thus the only 
finding is sinus tachycardia. In massive pulmonary embolism (PE), the evidence of 'right heart strain' may be seen (Rightward shift of the QRS axis, transient right bundle branch block, Qr pattern in V1, T-wave inversion in leads V1-3, SI QIII TIII pattern, P pulmonale), but these signs are nonspecific [18]. In combination with a 'high clinical pretest probability' or 'echocardiographic signs' of 'right ventricular dysfunction', accuracy of ECG to diagnose PE (Pulmonary embolism) may be improved. [19] Physicians should be aware about that, ECG-report may be 'entirely normal' in up to $20 \%$ of patients with PE resulting in a low sensitivity for the exclusion of the diagnosis. [20] Now a day, autonomic dysfunction in anxiety disorder, [21] even drowsiness [22] is assessed by ECG. ECG features are used widely in detection as the prediction input because it is a 'non-invasive' and 'radioactive free diagnostic' tool. [23] But as a single detector ECG is not enough for detecting PE or DVT.

Chest radiography. Chest radiography is the 'initial examination of choice' insuspected patients of pulmonary embolism (PE), especially in the outpatient setting. [24] Generally, radiographs are frequently 'abnormal' in the setting of pulmonary thromboembolism but, show nonspecific findings. Westermark [25] alluded to this fact inthe year of 1938, stated, "The roentgenologic diagnosis of 'embolism of the pulmonary artery' is verydifficult particularly, in cases 'without infarction'." In the year 1940, using 'postmortem radiographs', Hampton and Castleman [26] authored a radiologic-pathologic correlation where they described a 'pulmonary infarct' as a 'wedge-shaped opacity' touching the 'pleural surface', with a 'sharply convex medial margin'. In the year of 1991, Stein et al. [27] described, atelectasis and/or 'pulmonary parenchymal abnormalities' and 'pleural effusion' as the most common 'radiographic findings' in angiographically proven pulmonary embolismpatients. But finally, Milad et al. [24] claimed that, "thefindings and the aforementioned signs described by 'Hampton and Castleman'as well as by 'Fleischner' do not occur any more frequently in pulmonary embolism patients than in "patients without pulmonary embolism". So, chest radiography has some major limitation as a detector of PE and DVT.

Echocardiography. Transthoracic echocardiography (TE) rarely enables direct visualization of PE (Pulmonary embolus) [28] but may reveal thrombus floating "in transit" in the 'right atrium or ventricle'. By transesophageal echocardiography, it is also possible to visualize the massive emboli in 'central pulmonary ateries' [29]. In massive pulmonary embolism (PE), the right ventricle is usually dilated and hypokinetic, with the abnormal motion of the interventricular septum. [14] The finding of right ventricular dysfunction is non-specific [28] and certain conditions commonly confused with pulmonary embolism (such as chronic obstructive pulmonary disease exacerbations or cardiomyopathy) are also associated with abnormal right ventricular function. Although, direct echocardiographic visualization (EV) of intra-luminal thrombi in patients with suspected PE is an almost exceptional event and even when echocardiography provides only 'indirect signs' compatible with hemodynamic consequences of massive PE, it is helpful in excluding or suggesting alternative causes for any type of hemodynamic instability. Because, the right ventricle may present no dysfunction even in patients with massive PE (Pulmonary embolism), echocardiography should be considered an ancillary rather than a 'principal diagnostic test' for (PE) pulmonary embolism [30]. So in this method there are also many limitations. 
D-dimer test. D-dimer testing involves the conduction of a blood test to measure a substance that is released after a blood clot breaks up. A low or normal d-dimer test result indicates that only a minor amount of the substance released as the clot breaks up; thus, problems resulting from this blood clot are not likely. A higher-than-normal d-dimer level signals the potential for a blood clot issue to develop; d-dimer levels are often higher than normal in patients with abnormal blood clotting [31]. Elevations of D-dimer are non-specific; for instance, D-dimer is increased by aging, inflammation or cancer, and thus an abnormal result has a low positive predictive value. With respect to the role of d-dimer testing in the diagnosis of PE, a negative D-dimer result can assist in the exclusion of PE. The clinical probability estimate, determined by information from the patient's history and physical examination, can be assessed by either a formal numerical model,or an informed intuitive estimate [32]. The high negative likelihood ratio of these assays is sufficient to rule out $\mathrm{PE}$ in all patients; thus, these assays may be considered a "standalone" test for the exclusion of PE [33]. Because the "negative likelihood ratio' and 'predictive value of these tests' are not high enough to rule out pulmonary embolism in consecutive patients, a normal result must be combined with an additional assessment that classifies patients as having a lower pretest probability for pulmonary embolism. European Society of Cardiology guidelines state that, in $32-40 \%$ of patients with low to intermediate pretest clinical probability and normal D-dimer levels, PE can be safely excluded without further testing. [34] D-dimer testing as an assistant in the diagnosis of PE can be divided between two types of D-dimer assays, very highly sensitive and moderate-to-highly sensitive. Very highly sensitive D-dimer assays have a sensitivity for PE of around $98 \%$ or higher [36].

Lung ultrasound (LUS). Since its emergence approximately 15 years ago, [37] lung ultrasound technology has been increasingly used to complement conventional assessment methods and other imaging modalities of the lung in the diagnosis of PE [38]. Traditionally used to assess pleural effusions and masses, LUS has been revolutionized to image the pulmonary parenchyma, primarily as a point-of-care technique [37]. In general, the ultrasound imaging process has significant differences from radiographic imaging in which x-ray beams are used. LUS can be performed in any position and on the entire chest, laying the probe in the intercostal spaces and avoiding the ribs [37]. The probe is placed both longitudinally, perpendicular to the ribs, and obliquely along the intercostal spaces [37]. Ultrasound machines are lightweight, compact, easy to transport and robust, thus allowing multiple bedside examinations [39]. LUS is also not only easily available at bedside but, similar to MRI scans, can be performed with the absence of an ionizing radiation risk [39]. In addition to lower limb compressive venous ultrasonography and echocardiography, lung ultrasound can play an important role in the diagnosis of PE in selected patients' subgroups [40]. It can be safely used under conditions of both pregnancy and renal insufficiency, and can be highly useful as a bedside test for hemodynamically unstable patients [40].

Computed tomography (CT). As a fast and non-invasive technology, CT is often one of the first-line modalities for imaging of pulmonary circulation in patients with suspected PE [41]. CT can also reveal the extent of the PE, identify signs of right ventricular dysfunction, as well as provide alternative diagnoses. Basically, CT images 
are cross-sectional images of a specific area of a human body that allows doctors or surgeons to see the inside of a patient [42]. CT acquires images of the lung using a breath hold technique during the pulmonary arterial enhancement phase following the injection of intravenous contrast material. Similar to observations discovered through means of pulmonaryangiography, the PE would appear as a filling defect in the pulmonary artery as it becomes opaquer from thecontrast. Further advances in CT technology such as multidetector rows have allowed fora highly refined and detailed evaluation of the entire pulmonary vascular tree, and significant improvements in the detection of peripheral PE. Due to its noninvasive nature as well as its sensitivity and specificity, CT is currently considered the first line imaging tool for the evaluation of suspected PE.CT use for diagnosis of PE is increasing at a rapid pace due to large advances in technology that make CT user friendly for both the physician and patient [43]. Compared to conventional x-ray imaging procedures, CT exposes patients to higher doses of radiation. CT 16-array or greater delivers a higher absorbed dose $\left(8-20 \mathrm{mSv}^{3}\right)$ to breast tissue than conventional V/Qimaging $(0.6-3 \mathrm{mSv})$ [44]. These differences reflect variations in size and configuration of breast tissue, CT parameter settings, and the methods used to measure radiation dose. [44] An image [45] of CT is shown in Figure 1 for better concept.

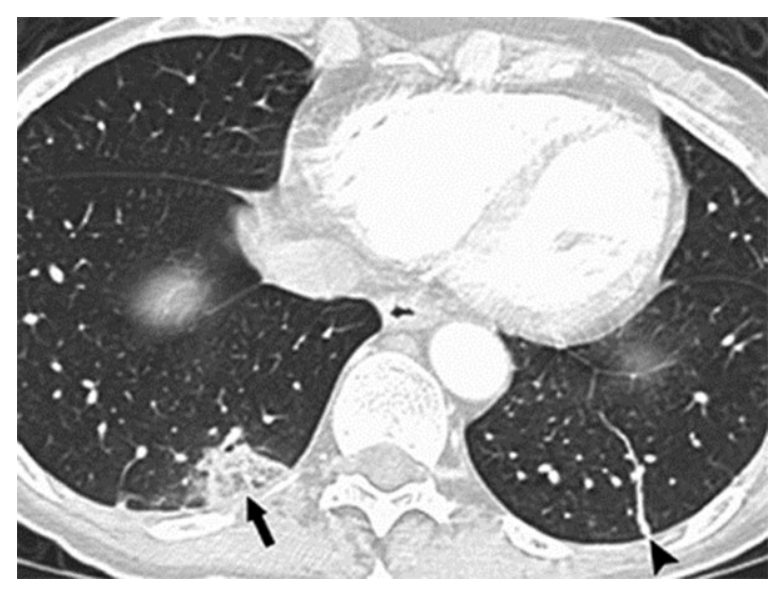

Fig. 1. Acute pulmonary embolism in a 58-year-old woman who presented with chest pain and dyspnea. CT scan shows an acute pulmonary embolus with ancillary findings of a peripheral wedge-shaped area of hyper attenuation in the lung (arrow), a finding that may represent an infarct, as well as a linear band (arrowhead)

Although data on the carcinogenic potential at relatively low dose $\mathrm{CT}$ imaging are lacking, the excess stochastic risk of fatal cancer induction in a standard person undergoing CT with the current effective dose of 3-6 mSv is $15-30$ excess deaths per 100,000 persons [46]. Various developments in CT imaging such as helical/spiral CT have enhanced the detection of small emboli as well as visuals of peripheral pulmonary arteries [47]. New technologies such as multi-detector row CT have also allowed for improvements towards optimizing contrast material delivery, and reducing radiation 
dose [47]. On the other hand, CT Venography (CTV) is an effective for diagnosis of DVT. Duplex ultrasound, including 'both gray-scale and Doppler imaging examination of the lower extremity venous system, has replaced conventional venography as the first-line diagnostic test for DVT, with reported sensitivity and specificity above $90 \%$ in symptomatic patients [48]. Ultrasound imaging can be performed at the bedside, does not involve ionizing radiation, is noninvasive, and is relatively inexpensive. The technical quality of the diagnosis de-pends on operator skill and evaluation of the "pelvic and calf veins' is limited [49]. On the other hand, combined CTPA/CTV fills this role, and the results of one component can be used to guide therapy when the complementary component is not diagnostic, increasing the overall cost-effectiveness [50]. The reported 'sensitivity and specificity' of CTV has been found between $89 \%-100 \%$ and $94 \%-100 \%$, respectively [51]. In one of the largest series, CTV was $97 \%$ sensitive and $100 \%$ specific for femoropopliteal DVT [52]. A more recent study using multislice CT showed a sensitivity of $100 \%$ and specificity of $97 \%$ and positive and negative predictive values of $92 \%$ and $100 \%$, respectively [53].

Magnetic resonance imaging (MRI). An Image processing concept called MRI can be used to visualize different structures of human body [54]. MRI techniques are used for diagnosing pulmonary embolism (PE) and deep vein thrombosis include MR angiography or MRA with/without contrast enhancement, 'MR perfusion imaging', and 'real-time MR'. MR angiography covers several advanced techniques like 'contrastenhanced high temporal resolution' and 'non-contrast- enhanced steady-state free procession (SSFP) sequences [55]. In 1984, findings from some experimental studies as well as some case reports suggested a role for MR imaging (MRI) in patients with pulmonary embolism (PE) [56]. After five years, in 1989, Hatabu and colleagues [57] become able to successfully depict the 'small peripheral pulmonary arteries' with an ECG-gated spin-echo acquisition in diastole and a 'gradient-recalled acquisition' in a steady state with breath holding. The proper combination of real-time MR imaging (RTMR), MR angiography, as well as MR perfusion imaging has been proved to increase the sensitivity for the detection of PE (Pulmonary embolism) [58]. The tremendous development of 'steady-state free precession sequences' also improved the use of MRI for the detection of PE (145) as, a quick sequence with a 'high contrastto-noise ratio' could be used to generate 'high-spatial-resolution' three-dimensional MR angiographic 'non- enhanced' images [59]. The successful association of 'cine steady-state free precession' imaging into clinical practice also ensured comprehensive evaluation of both the left and right ventricular functions [60]. On the other hand, 'real-time steady-state' free precession MRI provided an additional way to evaluate the 'central pulmonary vasculature' in patients with 'poor breath-holding capability' or with contraindicated to the use of gadolinium-based contrast material [61]. The proper development of 'phase-contrast imaging' in the decades of 1990 made it possible to quantify 'blood flow' in main, right, as well as left pulmonary arteries and to assess 'blood flow' patterns [62]. In fact, MR Pulmonary Angiography (MRPA) is another development of MRI. In the decade 1990, MRPA involved basically two-dimensional time-of-flight (TOF) methods with limited anatomic coverage [63]. 
A

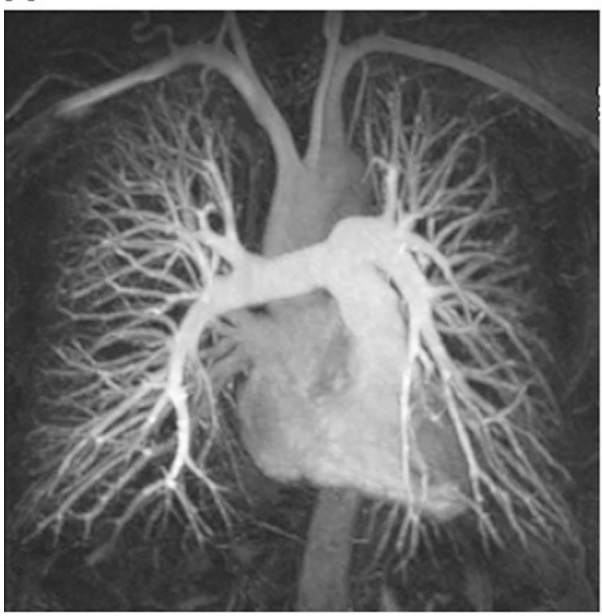

B

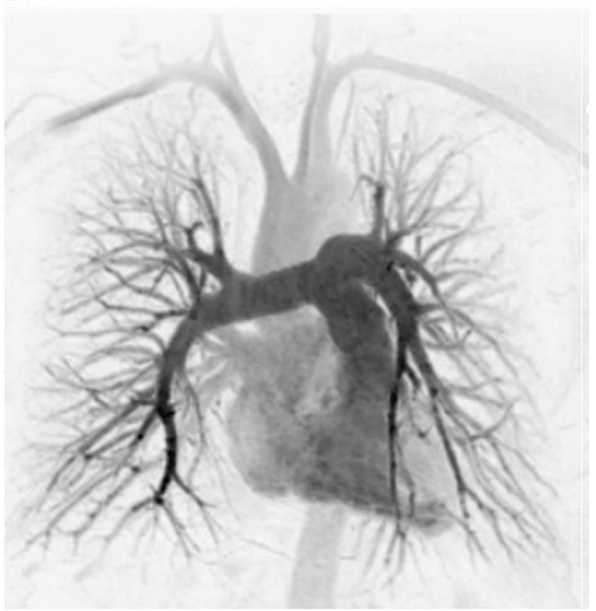

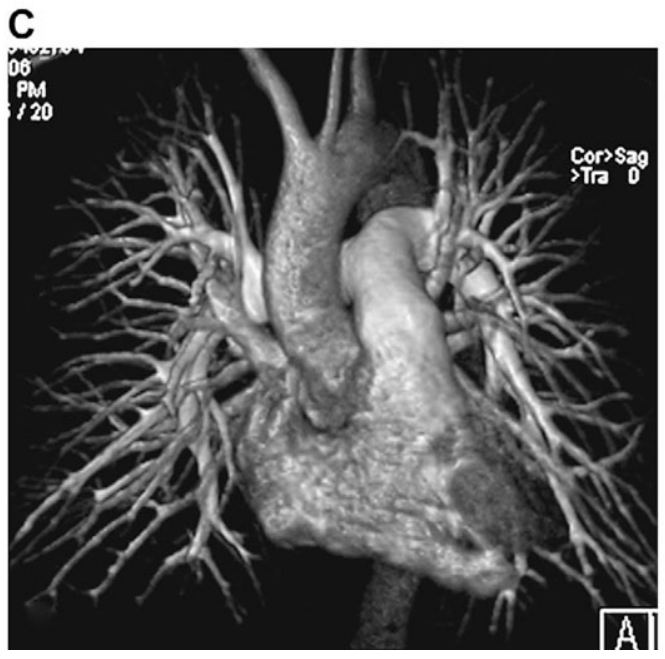

Fig. 2. High spatial resolution 3D CE MRA with parallel imaging with an acceleration factor of 2 in a 44-year-old male patient with shortness of breath and suspected PE. There was no evidence of filling defect or perfusional abnormality. Normal pulmonary arterial anatomy is demonstrated on the maximum intensity projection (MIP) (A), inverted MIP (B), and volume-rendered images $(\mathrm{C})[65]$

In this technic, breath-holding capability, 'registration artifacts', and 'poor differentiation of slow blood flow' from the thrombus also compromised the image quality [64]. The advent of prompt gradients and effecting reconstruction algorithms has made three-dimensional contrast MRA (MR angiography) feasible [63]. In Figure 2 there are some images of MRA [65] for assessing. Moreover, the technique of computed tomography pulmonary angiography (CTPA) is a faster, less complex, and less operator dependent than any other conventional pulmonary angiography, and has about the same 
frequency of technically insufficient examinations (about 5\%) [66]. In this method, the thorax can be scanned during the period of a single breath hold.Another one more advantage of computed tomography pulmonary angiography over scintigraphy is that by imaging the 'lung parenchyma' and great vessels, an alternative diagnosis can be made if PE is absent [67].

\subsection{Justification of using CT and MRI for DVT and PE}

Physicians and surgeons have been using CT and MRI for detecting DVT and PE for many years. CT scanners have the ability to acquire data for a slice typically between 0.5-4 seconds. In the first study to evaluate the accuracy of single-detector spiral CT angiography, Remy-Jardin et al. [68] reported a sensitivity of 100\% and specificity of $96 \%$ for pulmonary embolism in central pulmonary arteries, when compared with angiography as the diagnostic standard. CTPA studies using multidetector-row CTscanners have showed excellent sensitivity of $96-100 \%$ and specificity of $97-98 \%$ [69]. In that study, CTV performed as same to contrast venography, with the sensitivity of $100 \%$ and a specificity of $96 \%$. Some other studies were conducted on clinically suspected pulmonary embolism (PE) patients', generally without 'any sign and symptoms of the legs', in which the scan (CT) was extended to the legs. In a study it was found that, the estimated pooled sensitivity of 'CTV' was $96 \%$ with an 'estimated pooled specificity of $95 \%$ ' [70]. Of note, the heterogeneity between the studies was substantial.

In Table 1 the sensitivity and specificity of MRI techniques for Acute DVT \& PE Diagnosis [55] of a study is shown where the findings of many researchers are available.

Table 1. Sensitivity and specificity of MRI techniques for acute DVT \& PE diagnosis [55]

\begin{tabular}{|l|c|c|l|l|c|c|}
\hline \multicolumn{1}{|c|}{ First Author } & Year & $\mathbf{n}$ & \multicolumn{1}{c|}{ Patients } & \multicolumn{1}{c|}{ Technique } & Sensitivity & Specificity \\
\hline Erdman (56) & 1990 & 100 & Upper \& Low. ex. & Phase contrast & 90 & 100 \\
\hline Evans (58) & 1993 & 61 & Lower extremity & Phase con.\&MR & 100 & 100 \\
\hline Carpenter (75) & 1993 & 85 & Lower extremity & Time of flight & 100 & 96 \\
\hline Spritzer (57) & 1993 & 199 & Lower extremity & Time of flight & 97 & 98 \\
\hline Dupas (59) & 1995 & 25 & Pelvic vein & Time of flight & 100 & 98 \\
\hline \multicolumn{7}{|c|}{} \\
\hline
\end{tabular}

Note: *In case more sensitivity and specificity figures were mentioned in the article, we report the sensitivity and specificity of the proximal veins. 
From the late 1960s onwards, pulmonary angiography had been considered the "gold standard" imaging test for diagnosing PE [71]. Direct angiography allows for the visualization of thrombi as small as $1-2 \mathrm{~mm}$ within subsegmental arteries [72]. Today, a wide range of MRI techniques may be applied for diagnosis of VTD and PE [55]. Fraser et al. [73] included 101 patients suspected of DVT in their study that compared contrast venography with MRDTI (Magnetic resonance direct thrombus imaging). For gross DVT diagnosis, they found a good sensitivity of $94 \%$ to $96 \%$, regarding the qualifications of the observer. Specificity ranges were from $90 \%$ to $92 \%$. A meta-analysis showed a 'pooled estimate sensitivity' of $91.5 \%$ and specificity of $94.8 \%$ for DVT diagnosis by MRI [74] Compared to contrast venography, MRDTI has a sensitivity of $97 \%$ for femoropopliteal DVT and a specificity of 100\% [73] This benefit overcomes the limitations of other techniques that detect filling defects and surrogate markers of a thrombus [75] A prospective study on 511 patients with suspected pulmonary embolism (PE) demonstrated that, the sensitivity of CUS of the lower extremities for the presence of pulmonary embolism (PE) on CTPA was only 39\% with a specificity of $99 \%$ [76]. In a study, it has been found that, distal US has an even 'lower accuracy' for predicting pulmonary embolism (PE) with a sensitivity of $22 \%$ and a specificity of 94\% [77]. Gadolinium-enhanced MR Angiography is an effective and excellent noninvasive diagnostic method for PE because its 'sensitivity and specificity are high, as confirmed by a good review article and a meta-analysis, and it allows simultaneous study of DVT also [78]. A good advantage of MRA over multidetector 'CT angiography' is those images of ventilation may be obtained if noble gases, like helium 3 or xenon 129 and are used. Besides these, it has no contraindications and is very safe to use even during pregnancy [79]. Nothing that, MRA is more sensitive than Doppler ultrasound (DU) in detecting 'pelvic deep vein thrombosis (DVT) however [80]. As now MR technology improves and it becomes more readily available everywhere, the role of MRV and MRPA in assessing venous thromboembolic disease (VTD) may expand [63]. According to the positive references and findings we can consider CT and MRI as two time-tasted methods for detecting PE and DVT.

\section{Conclusion}

Day by day the prevalence of pulmonary embolism and deep vein thrombosis is increasing. For the patients of both the diseases are in need of prompt diagnosis. Considering the needed time there are some diagnostic methods like ECG or echocardiogram. But those methods are so effective as in needed. Some diagnostic technics like, ECG, echocardiogram, ultrasonography may be chosen as associated tests but for the clear concepts about the patient's condition of PE or DVT computed tomography (CT) and/ormagnetic resonance imaging (MRI) is a must. Moreover, combined use of CT and MRI may be considered as the method of choice for diagnosing PE and DVT. Besides these, the contentious trends of the development of CT and MRI also a justification of continuation of using those two methods in a wider range. 


\section{$5 \quad$ References}

[1] S.-H. Liu, J.-J. Wang, W. Chen, K.-L. Pan, and C.-H. Su, “An examination system to detect deep vein thrombosis of a lower limb using light reflection rheography," Sensors (Basel), vol. 21, no. 7, p. 2446, 2021. https://doi.org/10.3390/s21072446

[2] Y. M. Almotairi, "Relation between air travel and venous thromboembolism," J. med. sci. clin. res., vol. 05, no. 03, pp. 18393-18403, 2017. https://doi.org/10.18535/jmscr/v5i3.21

[3] J. H. Scurr, S. J. Machin, S. Bailey-King, I. J. Mackie, S. McDonald, and P. D. Smith, "Frequency and prevention of symptomless deep-vein thrombosis in long-haul flights: a randomised trial," Lancet, vol. 357, no. 9267, pp. 1485-1489, 2001. https://doi.org/10.1016/ $\underline{\text { S0140-6736(00)04645-6 }}$

[4] W. H. Geerts et al., "Prevention of venous thromboembolism," Chest, vol. 119, no. 1, pp. 132S-175S, 2001. https://doi.org/10.1378/chest.119.1 suppl.132S

[5] G. Macis, M. Salcuni, A. R. Cotroneo, G. Cina, and P. Marano, "Pulmonary thromboembolism and diagnostic imaging: integration of techniques and methods," Radiol. Med., vol. 92, no. $1-2$, pp. 63-71, 1996.

[6] S. Z. Goldhaber and H. Bounameaux, "Pulmonary embolism and deep vein thrombosis," Lancet, vol. 379, no. 9828, pp. 1835-1846, 2012. https://doi.org/10.1016/ S0140-6736(11)61904-1

[7] M. Cushman, "Epidemiology and risk factors for venous thrombosis," Semin. Hematol., vol. 44, no. 2, pp. 62-69, 2007. https://doi.org/10.1053/j.seminhematol.2007.02.004

[8] S. Z. Goldhaber, C. H. Hennekens, D. A. Evans, E. C. Newton, and J. J. Godleski, "Factors associated with correct antemortem diagnosis of major pulmonary embolism," Am. J. Med., vol. 73, no. 6, pp. 822-826, 1982. https://doi.org/10.1016/0002-9343(82)90764-1

[9] C. V. Pollack et al., "Clinical characteristics, management, and outcomes of patients diagnosed with acute pulmonary embolism in the emergency department: initial report of EMPEROR (Multicenter Emergency Medicine Pulmonary Embolism in the Real World Registry)," J. Am. Coll. Cardiol., vol. 57, no. 6, pp. 700-706, 2011. https://doi.org/10.1016/j. jacc.2010.05.071

[10] N. A. DeMonaco, Q. Dang, W. N. Kapoor, and M. V. Ragni, "Pulmonary embolism incidence is increasing with use of spiral computed tomography," Am. J. Med., vol. 121, no. 7, pp. 611-617, 2008. https://doi.org/10.1016/j.amjmed.2008.02.035

[11] B. H. Mbatchou Ngahane et al., "Epidemiology, clinical and paraclinical presentations of pulmonary embolism: A cross-sectional study in a sub-Saharan Africa setting," Open J. Respir. Dis., vol. 09, no. 03, pp. 89-99, 2019. https://doi.org/10.4236/ojrd.2019.93008

[12] Authors/Task Force Members et al., "2014 ESC Guidelines on the diagnosis and management of acute pulmonary embolism," Eur. Heart J., vol. 35, no. 43, pp. 3033-3080, 2014. https://doi.org/10.1093/eurheartj/ehu283

[13] J. A. Saleh and D. D. Alasia, “Acute pulmonary embolism: A review,” Niger. J. Med., vol. 16, no. 1, 2007. https://doi.org/10.4314/njm.v16i1.37274

[14] M. Riedel, "Diagnosing pulmonary embolism," Postgrad. Med. J., vol. 80, no. 944, pp. 309-319, 2004. https://doi.org/10.1136/pgmj.2003.007955

[15] P. D. Stein et al., "Clinical, laboratory, roentgenographic, and electrocardiographic findings in patients with acute pulmonary embolism and no pre-existing cardiac or pulmonary disease," Chest, vol. 100, no. 3, pp. 598-603, 1991. https://doi.org/10.1378/chest.100.3.598

[16] M. Pistolesi and M. Miniati, "Imaging techniques in treatment algorithms of pulmonary embolism," Eur. Respir. J., vol. 19, no. Supplement 35, pp. 28S - 39s, 2002. https://doi.org/ $\underline{10.1183 / 09031936.02 .00270602}$ 
[17] A. Perrier and H. Bounameaux, "Cost-effective diagnosis of deep vein thrombosis and pulmonary embolism," Thromb. Haemost., vol. 86, no. 1, pp. 475-487, 2001. https://doi. org/10.1055/s-0037-1616245

[18] N. Kucher, "QR in V1-an ECG sign associated with right ventricular strain and adverse clinical outcome in pulmonary embolism," Eur. Heart J., vol. 24, no. 12, pp. 1113-1119, 2003. https://doi.org/10.1016/S0195-668X(03)00132-5

[19] V. Rissanen et al., "Screening for postoperative pulmonary embolism on the basis of clinical symptomatology, routine electrocardiography and plain chest radiography," Acta Med. Scand., vol. 215, no. 1, pp. 13-19, 2009. https://doi.org/10.1111/j.0954-6820.1984.tb04964.x

[20] D. M. Weber and J. H. Phillips Jr, "A re-evaluation of electrocardiographs changes accompanying acute pulmonary embolism,” Am. J. Med. Sci., vol. 251, no. 4, pp. 381-398, 1966. https://doi.org/10.1097/00000441-196604000-00002

[21] A. S. George et al., "Predicting Autonomic dysfunction in anxiety disorder from ECG and respiratory signals using machine learning models," Int. J. Onl. Eng., vol. 17, no. 07, p. 143, 2021. https://doi.org/10.3991/ijoe.v17i07.22581

[22] D. Ribeiro, C. Teixeira, and A. Cardoso, "Web-based platform for training in biomedical signal processing and classification: The particular case of EEG-based drowsiness detection," Int. J. Online Eng., vol. 14, no. 03, p. 164, 2018. https://doi.org/10.3991/ijoe. v14i03.8193

[23] M. Z. Suboh, R. Jaafar, N. A. Nayan, and N. H. Harun, "ECG-based detection and prediction models of sudden cardiac death: Current performances and new perspectives on signal processing techniques," Int. J. Onl. Eng., vol. 15, no. 15, p. 110, 2019. https://doi.org/10.3991/ ijoe.v15i15.11688

[24] M. Yazdani et al., "Historical evolution of imaging techniques for the evaluation of pulmonary embolism:RSNA centennial article," Radiographics, vol. 35, no. 4, pp. 1245-1262, 2015. https://doi.org/10.1148/rg.2015140280

[25] N. Westermark, "On the roentgen diagnosis of lung embolism," Acta Radiol., vol. Original Series, 19, no. 4, pp. 357-372, 1938. https://doi.org/10.1177/028418513801900405

[26] B. C. AO Hampton, "Correlation of postmortem chest teleroentgenograms with autopsy findings with special reference to pulmonary embolism and infarction," Scienceopen.com. [Online]. Available: https://www.scienceopen.com/document?vid=30475dcc-e552-4893b944-d089583567ad. [Accessed: 27-Aug-2021].

[27] K. Malagari et al., "High-resolution CT findings in mild pulmonary fat embolism," Chest, vol. 123, no. 4, pp. 1196-1201, 2003. https://doi.org/10.1378/chest.123.4.1196

[28] M. Miniati et al., "Value of transthoracic echocardiography in the diagnosis of pulmonary embolism: results of a prospective study in unselected patients," Am. J. Med., vol. 110, no. 7, pp. 528-535, 2001. https://doi.org/10.1016/S0002-9343(01)00693-3

[29] P. Pruszczyk, A. Torbicki, A. Kuch-Wocial, M. Szulc, and R. Pacho, "Diagnostic value of transoesophageal echocardiography in suspected haemodynamically significant pulmonary embolism," Heart, vol. 85, no. 6, pp. 628-634, 2001. https://doi.org/10.1136/heart.85.6.628

[30] M. Riedel, "Diagnosing pulmonary embolism," Postgrad. Med. J., vol. 80, no. 944, pp. 309-319, 2004. Available: https://pmj.bmj.com/content/postgradmedj/80/944/309.full. pdf [Accessed: 27-Aug-2021]. https://doi.org/10.1136/pgmj.2003.007955

[31] M. Gurram and S. Pulivarthi, "Effectiveness of D-dimer as a screening test for venous thromboembolism: An update," N. Am. J. Med. Sci., vol. 6, no. 10, p. 491, 2014. https://doi. org/10.4103/1947-2714.143278

[32] P. D. Stein et al., "Diagnostic pathways in acute pulmonary embolism: recommendations of the PIOPED II Investigators," Radiology, vol. 242, no. 1, pp. 15-21, 2007. https://doi. org/10.1148/radiol.2421060971 
[33] A. Perrier et al., "Non-invasive diagnosis of venous thromboembolism in outpatients," Lancet, vol. 353, no. 9148, pp. 190-195, 1999. https://doi.org/10.1016/S0140-6736(98)05248-9

[34] S. V. Konstantinides et al., "Corrigendum to: 2014 ESC Guidelines on the diagnosis and management of acute pulmonary embolism," Eur. Heart J., vol. 36, no. 39, p. 2642, 2015. https://doi.org/10.1093/eurheartj/ehu479

[35] "Pulmonary embolism research," Nih.gov. [Online]. Available: https://www.nhlbi.nih.gov/ events/2011/pulmonary-embolism-research. [Accessed: 27-Aug-2021].

[36] C. Kearon, "Diagnosis of pulmonary embolism," CMAJ, vol. 168, no. 2, pp. 183-194, 2003.

[37] L. Gargani and G. Volpicelli, "How I do it: lung ultrasound," Cardiovasc. Ultrasound, vol. 12, no. 1, p. 25, 2014. https://doi.org/10.1186/1476-7120-12-25

[38] A. Reissig and C. Kroegel, "Transthoracic ultrasound of lung and pleura in the diagnosis of pulmonary embolism: a novel non-invasive bedside approach," Respiration, vol. 70, no. 5, pp. 441-452, 2003. https://doi.org/10.1159/000074195

[39] B. Bouhemad, M. Zhang, Q. Lu, and J.-J. Rouby, "Clinical review: Bedside lung ultrasound in critical care practice," Crit. Care, vol. 11, no. 1, p. 205, 2007. https://doi.org/10.1186/ $\underline{\operatorname{cc} 5668}$

[40] A. Squizzato, L. Galli, and V. E. A. Gerdes, "Point-of-care ultrasound in the diagnosis of pulmonary embolism," Crit. Ultrasound J., vol. 7, no. 1, p. 7, 2015. https://doi.org/10.1186/ s13089-015-0025-5

[41] H. Doğan, A. de Roos, J. Geleijins, M. V. Huisman, and L. J. M. Kroft, "The role of computed tomography in the diagnosis of acute and chronic pulmonary embolism," Diagn. Interv. Radiol., vol. 21, no. 4, pp. 307-316, 2015. https://doi.org/10.5152/dir.2015.14403

[42] A. Kalidindi, P. L. Kompalli, S. Bandi, and S. R. R. Anugu, "CT image classification of human brain using deep learning," Int. J. Onl. Eng., vol. 17, no. 01, p. 51, 2021. https://doi. org/10.3991/ijoe.v17i01.18565

[43] D. J. Brenner and E. J. Hall. "Computed tomography - an increasing source of radi-ation exposure." New England journal of medicine, vol. 357.22, p. 2277-2284, 2007. https://doi. org/10.1056/NEJMra072149

[44] G. P. Schembri, A. E. Miller, and R. Smart, "Radiation dosimetry and safety issues in the investiga-tion of pulmonary embolism." Semin Nucl Med, vol. 40(6), p. 442-454, 2010. https://doi.org/10.1053/j.semnuclmed.2010.07.007

[45] C. Wittram, M. M. Maher, A. J. Yoo, M. K. Kalra, J.-A. O. Shepard, and T. C. McLoud, "CT angiography of pulmonary embolism: diagnostic criteria and causes of misdiagnosis," Radiographics, vol. 24, no. 5, pp. 1219-1238, 2004. https://doi.org/10.1148/rg.245045008

[46] ICRP, "The 2007 Recommendations of the international commission on Radiological Protection. ICRP publication 103," Ann. ICRP, vol. 37, no. 2-4, pp. 1-332, 2007.

[47] J. R. Mayo, "Radiation dose issues in longitudinal studies involving computed tomography," Proc. Am. Thorac. Soc., vol. 5, no. 9, pp. 934-939, 2008. https://doi.org/10.1513/ pats.200808-079QC

[48] R. H. White, J. P. McGahan, M. M. Daschbach, and R. P. Hartling, "Diagnosis of deep-vein thrombosis using duplex ultrasound," Ann. Intern. Med., vol. 111, no. 4, pp. 297-304, 1989. https://doi.org/10.7326/0003-4819-111-4-297

[49] T. W. Wakefield and L. J. Greenfield, "Diagnostic approaches and surgical treatment of deep venous thrombosis and pulmonary embolism," Hematol. Oncol. Clin. North Am., vol. 7, no. 6, pp. 1251-1267, 1993. https://doi.org/10.1016/S0889-8588(18)30206-5

[50] L. R. Goodman, "1999 plenary session: Friday imaging symposium: CT diagnosis of pulmonary embolism and deep venous thrombosis," Radiographics, vol. 20, no. 4, pp. 1201-1205, 2000. https://doi.org/10.1148/radiographics.20.4.g00j1161201 
[51] K. M. Duwe, M. Shiau, N. E. Budorick, J. H. Austin, and Y. M. Berkmen, "Evaluation of the lower extremity veins in patients with suspected pulmonary embolism: a retrospective comparison of helical CT venography and sonography. 2000 ARRS Executive Council Award I. American Roentgen Ray Society: A retrospective comparison of helical CT venography and sonography," AJR Am. J. Roentgenol., vol. 175, no. 6, pp. 1525-1531, 2000. https://doi. org/10.2214/ajr.175.6.1751525

[52] P. A. Loud, D. S. Katz, D. A. Bruce, D. L. Klippenstein, and Z. D. Grossman, "Deep venous thrombosis with suspected pulmonary embolism: detection with combined CT venography and pulmonary angiography," Radiology, vol. 219, no. 2, pp. 498-502, 2001. https://doi. org/10.1148/radiology.219.2.r01ma26498

[53] P. G. C. Begemann et al., "Evaluation of the deep venous system in patients with suspected pulmonary embolism with multi-detector CT: a prospective study in comparison to Doppler sonography," J. Comput. Assist. Tomogr., vol. 27, no. 3, pp. 399-409, 2003. https://doi. org/10.1097/00004728-200305000-00017

[54] A. Kalidindi, P. L. Kompalli, S. Bandi, and S. R. R. Anugu, "CT image classification of human brain using deep learning," Int. J. Onl. Eng., vol. 17, no. 01, p. 51, 2021. https://doi. org/10.3991/ijoe.v17i01.18565

[55] K. van Langevelde, M. Tan, A. Šrámek, M. V. Huisman, and A. de Roos, "Magnetic resonance imaging and computed tomography developments in imaging of venous thromboembolism," J. Magn. Reson. Imaging, vol. 32, no. 6, pp. 1302-1312, 2010. https:// doi.org/10.1002/jmri.22379

[56] G. Gamsu, M. Hirji, E. H. Moore, W. R. Webb, and A. Brito, "Experimental pulmonary emboli detected using magnetic resonance," Radiology, vol. 153, no. 2, pp. 467-470, 1984. https://doi.org/10.1148/radiology.153.2.6484179

[57] H. Hatabu, W. B. Gefter, H. Y. Kressel, L. Axel, and R. E. Lenkinski, "Pulmonary vasculature: high-resolution MR imaging. Work in progress," Radiology, vol. 171, no. 2, pp. 391-395, 1989. https://doi.org/10.1148/radiology.171.2.2704803

[58] A. Kluge, W. Luboldt, and G. Bachmann, "Acute pulmonary embolism to the subsegmental level: diagnostic accuracy of three MRI techniques compared with 16-MDCT," AJR Am.J. Roentgenol., vol. 187, no. 1, pp. W7-14, 2006. https://doi.org/10.2214/AJR.04.1814

[59] M. S. Krishnam et al., "Noncontrast 3D steady-state free-precession magnetic resonance angiography of the whole chest using nonselective radiofrequency excitation over a large field of view: Comparison with single-phase 3D contrast-enhanced magnetic resonance angiography," Invest. Radiol., vol. 43, no. 6, pp. 411-420, 2008. https://doi.org/10.1097/ RLI.0b013e3181690179

[60] K. Alfakih, S. Plein, T. Bloomer, T. Jones, J. Ridgway, and M. Sivananthan, "Comparison of right ventricular volume measurements between axial and short axis orientation using steady-state free precession magnetic resonance imaging," J. Magn. Reson. Imaging, vol. 18, no. 1, pp. 25-32, 2003. https://doi.org/10.1002/jmri.10329

[61] A. Kluge, C. Müller, J. Hansel, T. Gerriets, and G. Bachmann, "Real-time MR with TrueFISP for the detection of acute pulmonary embolism: Initial clinical experience," Clin. Imaging, vol. 28, no. 5, pp. 387-388, 2004. https://doi.org/10.1016/j.clinimag.2004.06.012

[62] V. L. Morgan, R. J. Roselli, and C. H. Lorenz, "Normal three-dimensional pulmonary artery flow determined by phase contrast magnetic resonance imaging," Ann. Biomed. Eng., vol. 26, no. 4, pp. 557-566, 1998. https://doi.org/10.1114/1.125

[63] J. P. Kanne and T. A. Lalani, "Role of computed tomography and magnetic resonance imaging for deep venous thrombosis and pulmonary embolism," Circulation, vol. 109, no. 12 Suppl 1, pp. I15-21, 2004. https://doi.org/10.1161/01.CIR.0000122871.86662.72 
[64] J. F. Meaney, L. O. Johansson, H. Ahlstrom, and M. R. Prince, "Pulmonary magnetic resonance angiography," J. Magn. Reson. Imaging, vol. 10, no. 3, pp. 326-338, 1999. https://doi. org/10.1002/(SICI)1522-2586(199909)10:3<326::AID-JMRI15>3.0.CO;2-L

[65] E. M. Hecht and A. Rosenkrantz, "Pulmonary MR angiography techniques and applications," Magn. Reson. Imaging Clin. N. Am., vol. 17, no. 1, pp. 101-131, 2009. https://doi. org/10.1016/j.mric.2009.01.001

[66] Y. Ruiz et al., "Prospective comparison of helical CT with angiography in pulmonary embolism: global and selective vascular territory analysis. Interobserver agreement," Eur. Radiol., vol. 13, no. 4, pp. 823-829, 2003. https://doi.org/10.1007/s00330-002-1588-7

[67] M. J. L. van Strijen et al., "Single-detector helical computed tomography as the primary diagnostic test in suspected pulmonary embolism: a multicenter clinical management study of 510 patients," Ann. Intern. Med., vol. 138, no. 4, pp. 307-314, 2003. https://doi. org/10.7326/0003-4819-138-4-200302180-00009

[68] M. Remy-Jardin, J. Remy, L. Wattinne, and F. Giraud, "Central pulmonary thromboembolism: diagnosis with spiral volumetric CT with the single-breath-hold technique--comparison with pulmonary angiography," Radiology, vol. 185, no. 2, pp. 381-387, 1992. https://doi. org/10.1148/radiology.185.2.1410342

[69] R. Quiroz et al., "Clinical validity of a negative computed tomography scan in patients with suspected pulmonary embolism: a systematic review: A systematic review," JAMA, vol. 293, no. 16, pp. 2012-2017, 2005. https://doi.org/10.1001/jama.293.16.2012

[70] S. M. Thomas, S. W. Goodacre, F. C. Sampson, and E. J. R. van Beek, "Diagnostic value of CT for deep vein thrombosis: results of a systematic review and meta-analysis," Clin. Radiol., vol. 63, no. 3, pp. 299-304, 2008. https://doi.org/10.1016/j.crad.2007.09.010

[71] G. A. Miller, G. C. Sutton, I. H. Kerr, R. V. Gibson, and M. Honey, "Comparison of streptokinase and heparin in treatment of isolated acute massive pulmonary embolism," Br. Heart J., vol. 33, no. 4, p. 616, 1971.

[72] M. Wolfe, "Pulmonary embolic disease: Diagnosis, pathophysiologic aspects, and treatment with thrombolytic therapy," Curr. Probl. Cardiol., vol. 18, no. 10, pp. 590-633, 1993. https://doi.org/10.1016/0146-2806(93)90006-N

[73] D. G. W. Fraser, A. R. Moody, P. S. Morgan, A. L. Martel, and I. Davidson, "Diagnosis of lower-limb deep venous thrombosis: a prospective blinded study of magnetic resonance direct thrombus imaging," ACC Curr. J. Rev., vol. 11, no. 4, pp. 54-55, 2002. https://doi. org/10.1016/S1062-1458(02)00716-X

[74] F. C. Sampson, S. W. Goodacre, S. M. Thomas, and E. J. R. van Beek, "The accuracy of MRI in diagnosis of suspected deep vein thrombosis: systematic review and meta-analysis," Eur. Radiol., vol. 17, no. 1, pp. 175-181, 2007. https://doi.org/10.1007/s00330-006-0178-5

[75] J. Kelly, B. J. Hunt, and A. Moody, "Magnetic resonance direct thrombus imaging: a novel technique for imaging venous thromboemboli," Thromb. Haemost., vol. 89, no. 5, pp. 773-782, 2003. https://doi.org/10.1055/s-0037-1613461

[76] G. Le Gal et al., "A positive compression ultrasonography of the lower limb veins is highly predictive of pulmonary embolism on computed tomography in suspected patients," Thromb. Haemost., vol. 95, no. 6, pp. 963-966, 2006. https://doi.org/10.1160/TH06-03-0158

[77] M. Righini et al., "Complete venous ultrasound in outpatients with suspected pulmonary embolism," J. Thromb. Haemost., vol. 7, no. 3, pp. 406-412, 2009. https://doi. org/10.1111/j.1538-7836.2008.03264.x

[78] P. D. Stein et al., "Gadolinium-enhanced magnetic resonance angiography for pulmonary embolism: a multicenter prospective study (PIOPED III): A multicenter prospective study (PIOPED III)," Ann. Intern. Med., vol. 152, no. 7, pp. 434-43, W142-3, 2010. https://doi. org/10.7326/0003-4819-152-7-201004060-00008 
[79] H. Hatabu, "MR pulmonary angiography and perfusion imaging: recent advances," Semin. Ultrasound CT MR, vol. 18, no. 5, pp. 349-361, 1997. https://doi.org/10.1016/ S0887-2171(97)90012-3

[80] A. R. Moody, "Magnetic resonance direct thrombus imaging," J. Thromb. Haemost., vol. 1, no. 7, pp. 1403-1409, 2003. https://doi.org/10.1046/j.1538-7836.2003.00333.x

\section{Authors}

Petro Bodnar, PhD, MD, Associate Professor, L.Ya. Kovalchuk Department of Surgery No.1, Urology, Minimally Invasive Surgery I. Horbachevsky Ternopil National Medical University, Ternopil, Ukraine ORCID: 0000-0001-9285-6516, Researcher ID: C-7601-2016, Scopus Author ID: 57203426626 (email: Bodnarpj@tdmu.edu.ua). https://drive.google.com/file/d/1PllZhwB9TzG5uKx3dgNcLc6faMzpxC_k/view; https://drive.google.com/file/d/102f8PlWvJiBPQc1DTOef1MNLYfi70e5H/view

Yaroslav Bodnar, PhD, MD, Professor Department of Pathologic Anatomy, Autopsy Course and Forensic Pathology, I. Horbachevsky Ternopil National Medical University, Ternopil, Ukraine, ORCID: 0000-0002-5507-5363, Researcher ID: R-64182016 (email: bodnarya@tdmu.edu.ua) https://pat-anatomy.tdmu.edu.ua/struktura/ bodnar-aroslav-aroslavovic

Tetiana Bodnar, PhD, MD, Assistant Professor, L.Ya. Kovalchuk Department of Surgery No.1, Urology, Minimally Invasive Surgery, I. Horbachevsky Ternopil National Medical University, Ternopil, Ukraine, ORCID: 0000-0002-5507-5363, ResearcherID:R-6418-2016(email:bodnartv@tdmu.edu.ua)https://www.researchgate.net/profile/Tetiana-Bodnar, https://en.surgery1.tdmu.edu.ua/struktura/korotkabiograficna-dovidka-4, https://en.surgery1.tdmu.edu.ua/struktura/naukovi-publikaciieas-bodnar-t-v

Liudmyla Bodnar, PhD, MD, Associate Professor, Internal Medicine No.3, I. Horbachevsky Ternopil National Medical University, Ternopil, Ukraine ORCID: 00000001-9173-930X, Researcher ID: B-2584-2017, https://www.researchgate.net/profile/ Lyudmyla-Bodnar-2 (email: Bodnarpj@tdmu.edu.ua)

Dymytriy Hvalyboha, PhD, Surgery No.2, I. Horbachevsky Ternopil National Medical University, Ternopil, Ukraine ORCID 0000-0003-0168-0848 (email: Bodnarpj@,tdmu.edu.ua)

Article submitted 2021-08-27. Resubmitted 2021-10-08. Final acceptance 2021-10-08. Final version published as submitted by the authors. 